\title{
Information Needs of Black Prostate Cancer Patients Receiving Treatment Within the South African Public Healthcare System
}

\author{
Melissa Taljaard - Germaine T. Lovric - Aviwe M. Makenzi • \\ Prudence Kawinga
}

Received: April 28, 2020 / Published online: August 27, 2020

(C) The Author(s) 2020

\begin{abstract}
Introduction: Prostate cancer is the leading cancer type in black South African men. The South African public healthcare sector serves more than $84 \%$ of the population, which includes many of these men. Previous evidence suggests that patients' information needs are influenced by culture. No studies could be found that explored the information needs of black men diagnosed with prostate cancer in a developing country from the patients' perspectives. Therefore, this study set out to investigate the information needs of black men diagnosed with prostate cancer in a South African public healthcare setting.
\end{abstract}

Methods: Nine participants who had completed a radical course of external beam radiation therapy for prostate cancer were interviewed. These participants had gained experience from their cancer journey in this setting and could therefore provide information-rich perspectives about their information needs from the time of diagnosis to end of

Digital Features To view digital features for this article go to https://doi.org/10.6084/m9.figshare.12783626.

M. Taljaard · G. T. Lovric ( $\square)$ - A. M. Makenzi · P. Kawinga

Department of Radiography, Faculty of Health

Sciences, University of Pretoria, Pretoria 0001, South

Africa

e-mail: germaine.mathurine@up.ac.za treatment. Interviews were conducted in the participants' preferred language, with three interviews conducted in Zulu with an English translator.

Results: Important themes that emerged included patients' desire to receive more information regarding what was happening in the diagnosis stage, the implications of having prostate cancer and the origin of their symptoms. The participants expressed a need to understand the potential side effects of radiation therapy, the reason for bladder filling and on-treatment set-up imaging verification. Participants also wanted to know how they should care for themselves and adjust their lifestyles, and required more information about follow-up tests and appointments.

Conclusion: In South Africa, black men with prostate cancer expressed the need for more information about the implications of a prostate cancer diagnosis, the reasons for these treatments and what they were expected to do. They also require information about where to go and what will happen in the different parts of the healthcare system with regard to the diagnosis and treatment of the prostate cancer. Communities should also be educated about cancer to avoid misconceptions. In South Africa, healthcare workers should consider the life-worlds of black men in the public healthcare system when attending to their information needs. 
Keywords: Information needs; Prostate cancer; Public healthcare system

\section{Key Summary Points}

Prostate cancer is one on the most prevalent cancers in men, but no studies have been conducted to explore the information needs of black men diagnosed with prostate cancer in a developing country.

This study explored the specific information needs of black men diagnosed with prostate cancer in a South African public hospital, as previous evidence suggests that culture has been found to play a central role in patient's information needs.

The study concluded that more information is needed regarding the implications of a prostate cancer diagnosis, the process involved, treatments, the reasons for these treatments and what is expected of patients diagnosed with prostate cancer.

Healthcare workers should consider the life-worlds of black men in the public healthcare system when attending to their information needs, and communities should be educated about cancer to avoid misconceptions.

\section{INTRODUCTION}

According to the 2018 GLOBOCAN report, the incidence of prostate cancer has risen to 64.1 per 100,000 people in the Southern African region [1]. Prostate cancer in South Africa is unfortunately underrepresented, as there is no national registry for this cancer type [2]. Separate studies conducted in public sector hospitals in Bloemfontein and Cape Town, South Africa both reported that the black patients in their study groups tended to present with higher prostate-specific antigen (PSA) values, and locally advanced and high-grade disease [3, 4].

Most patients in South Africa are likely to access treatment through the public healthcare system, which serves $84 \%$ of the population [5]. The hierarchy of the South African public healthcare referral system is complex and consists of the following lines of referral: primary healthcare facility (clinic/community health centre), district hospital, regional hospital, and lastly a tertiary hospital [6]. Patients accessing the South African public health system are faced with system constraints such as shortages of specialist urologists and delays because of overcrowding in the system [2]. The public healthcare sector radiation therapy departments are located in certain central and tertiary hospitals that are found in the large cities [7]. Patients are faced with commuting and accommodation problems when they need to undergo cancer treatments.

Patients diagnosed with prostate cancer go through various treatment pathways, including prostatectomy (surgery), brachytherapy (internal radiation therapy), androgen deprivation therapy (ADT) (also referred to as hormone therapy), watchful waiting (monitoring) and external beam radiation therapy (EBRT) [8]. In the public tertiary hospital of our study, patients with advanced intermediate- or highgrade adenocarcinoma of the prostate received a minimum of two doses of ADT before commencing EBRT. In some cases three doses might be given when there are delays in receiving EBRT due to problems with availability of linear accelerator (machine administering EBRT) treatment booking slots. The hormonal treatment consists of three monthly injections of $10.8 \mathrm{mg}$ of Zoladex ${ }^{\circledR}$ and continues for 24 to 36 months. The patients received 3-D conformal EBRT consisting of 37 fractions to a total dose of $74 \mathrm{~Gy}$, and therefore received radiation treatments for 7 to 8 weeks.

For cancer patients, a critical element of their journey is receiving information from healthcare professionals relevant to their needs $[9,10]$. Many studies have assessed the core information needs of prostate cancer patients to guide professionals in how they communicate with their patients. These studies were largely 
quantitative, using questionnaires developed in first-world settings [11-16]. Studies have suggested that culture may influence the information needs of cancer patients $[17,18]$. There is some evidence that cancer patients receiving treatment in the South African public healthcare system receive inconsistent information throughout their journey [19]. No studies have assessed the information needs of black men in South Africa diagnosed with prostate cancer. In this qualitative study, we explored the perceptions of black men diagnosed with prostate cancer in the public healthcare sector regarding their information needs.

\section{METHODS}

We followed an exploratory-descriptive qualitative methodology to discover and understand the information needs of men diagnosed with prostate cancer [20]. This approach allowed us to gain an in-depth understanding of participants' literal descriptions and the proxy interpretations of the researchers [21].

\section{Sampling}

A combination of purposive and convenience sampling methods were used to select patients who were attending a public sector radiation oncology department for participation in this study. A research member consulted the patient files to determine whether the patients met the inclusion criteria, i.e. they had a positive histology of prostatic adenocarcinoma, had an Eastern Cooperative Oncology Group (ECOG) performance status of 0 to 3 [8] and were at the end of the course of radical EBRT localised to the prostate. The patients' tumour stage and risk profiles were not taken into consideration upon selection. We assumed that these patients would be able to provide information-rich data, because they had traversed the healthcare system from diagnosis to end of treatment.

\section{Data Collection}

The study participants signed an informed consent form, after a comprehensive explanation and information leaflet was given to them. No participant refused to participate and no attrition from the study occurred. The National Health Research Department gave permission to interview the patients in this public hospital. The University of Pretoria Health Sciences Ethics Committee approved the study, with Ethics Reference No. 602/2018. Informed consent was obtained from all participants, and the study was conducted in accordance with the Declaration of Helsinki.

Semi-structured interviews were conducted in the participant's preferred language. Three of the nine participants requested that their interviews be conducted in Zulu. These interviews were conducted with the aid of a translator who was bilingual in Zulu and English. The translator signed a confidentiality agreement. Participants were interviewed in a quiet office in the radiation oncology department, directly after finishing their last radiation treatment. The office was arranged by the researchers to ensure the comfort and privacy of the participants, with the interviews captured on a digital recorder.

The youngest participant was 46 years old, whilst the oldest was 76 years. All participants were of a black race and had an ECOG performance status of 1 . All had locally advanced prostate cancer, with two having low-grade, three having intermediate-grade and four having high-grade disease. All had above-normal PSA values ranging from 265 to $1486 \mathrm{ng} / \mathrm{ml}$. Three of the participants had to travel from another province to receive treatment at this tertiary hospital. Most participants residing in the province where the tertiary hospital was located lived in townships a considerable distance from the hospital. 
The interviews commenced with an icebreaker question, asking, "What are you feeling now that you are completing your radiation treatment?" The interviews were guided by the central open-ended question posed to each of the participants: "Now that you have come through being diagnosed and treated for your prostate cancer, please can you share what information you would have liked to and needed to know about the cancer and treatments"?

The longest interview lasted $24 \mathrm{~min} 40 \mathrm{~s}$, while the shortest interview was $2 \min 35 \mathrm{~s}$, with the average duration of the interviews being $131 / 2$ minutes.

The researchers transcribed the interviews verbatim. One of the researchers, who is bilingual in English and Zulu, verified the translator's interpretations of the Zulu interviews. The translator had provided highly accurate versions of what the participants had said. The three researchers rotated the role of interviewer, i.e. if researcher 1 conducted the interview, researcher 2 would transcribe, and researcher 3 would review the document to ensure accuracy of the transcription.

\section{Data Analysis}

All three researchers immersed themselves in the transcripts when considering the research question. Using a process of inductive content analysis, they read the participants' narratives to make sense of what was being said [22]. The researchers independently coded the phrases and words in the transcripts using a combination of in vivo and process coding [23, 24]. They then compared their independent codes until they agreed on the final codes. These codes were placed into categories. Categories and themes were developed using pattern coding as secondcycle coding $[23,24]$.

\section{RESULTS}

Participants shared their experiences and expectations of their cancer care journey that shaped their information needs. The duration of the interviews was dependent on the extent to which the participants could or wanted to share their thoughts with the interviewers. An outlier in this study was participant 3 , who spoke only for $2 \mathrm{~min} 35 \mathrm{~s}$, and indicated that he had received all the information that he needed.

"I was properly informed. The doctor was very kind. She told me exactly what is going to happen."

He was fortunately diagnosed and treated at the same hospital.

"I did everything here [at the tertiary hospital]."

Due to the way that participant 3 was managed, he was satisfied with his oncology experience.

"I'm very happy, ja [yes], very happy."

The rest of the participants had gone through a more complex healthcare pathway to be diagnosed and receive their treatments.

Participants explained that they had received information about the cancer and its treatment from various sources. Participants mentioned that they took the onus on themselves to obtain and understand information about prostate cancer and treatments. One participant explained:

"So one should have just maybe enquired deeper to find out. But, I think, on the other hand I think I, I should have, should have sought that information for myself. We must also play our part to say, you know, as a patient to find [out] what this is all about. How does it happen?" (Participant 7)

Some participants turned to trusted "others" to gain information, and to help them source information from the internet and printed information. Examples can be seen in the following quotes by three different participants:

"A mayor in $\mathrm{M}$ (low-income urban settlement) is now late, he tell me about cancer boost so I Google it. I find it in my phone, that there is something that can cure this cancer." (Participant 4) 
Table 1 Typography of themes, sub-themes and categories related to the information needs of men with prostate cancer

\begin{tabular}{|c|c|c|}
\hline Theme & Sub-themes & Categories \\
\hline \multirow[t]{10}{*}{$\begin{array}{l}\text { Personalised information to answer worrying } \\
\text { questions }\end{array}$} & \multirow[t]{2}{*}{$\begin{array}{l}\text { What's happening in the } \\
\text { beginning? }\end{array}$} & $\begin{array}{l}\text { Blood testing in primary health } \\
\text { clinics }\end{array}$ \\
\hline & & $\begin{array}{l}\text { Reasons for being sent between } \\
\text { hospitals }\end{array}$ \\
\hline & \multirow[t]{5}{*}{$\begin{array}{l}\text { What does this mean to my } \\
\text { life and me? }\end{array}$} & $\begin{array}{l}\text { What is cancer, and specifically } \\
\text { prostate cancer? }\end{array}$ \\
\hline & & Where is it in relation to the body? \\
\hline & & How serious is it? \\
\hline & & Why the treatments? \\
\hline & & What to do and not to do? \\
\hline & \multirow[t]{3}{*}{ The radiation treatments? } & About the treatment? \\
\hline & & $\begin{array}{l}\text { How will the radiation affect the } \\
\text { body? }\end{array}$ \\
\hline & & What happens next? \\
\hline \multirow{4}{*}{$\begin{array}{l}\text { Information needs to address the general } \\
\text { connotations of prostate cancer }\end{array}$} & \multirow[t]{3}{*}{ Shame in the illness } & Blame for not understanding \\
\hline & & Being seen "as a cancer" \\
\hline & & "Man issues" \\
\hline & Message to other men & "Getting tested" \\
\hline
\end{tabular}

"Luckily I have a friend who works for a doctor. And I thought I'd ask him questions. We play, we play golf together. And I say to him, 'What is this all about?' Then he explained. For me, I'm lucky he explained." (Participant 7)

"I mean, you know we have libraries, we have, you know, the information is there. There are books in the house that one can read or look for a book and read. You know, and I have those books at home which I am going to read also. Because this is not the end of the road. Now, if I still want to maybe improve myself, I must go to those books, read about this." (Participant 8)

Despite finding information on their own, participants still had concerning questions for which they required personalised answers. Participants also posed broader questions related to prostate cancer in general. The following themes, sub-themes and associated categories emerged from the participants' narratives are presented here and summarised in Table 1.

\section{THEME 1: PERSONALISED INFORMATION TO ANSWER WORRYING QUESTIONS}

Participants expressed a need for personalised answers to the following "most worrying" questions:

\section{What's Happening in the Beginning?}

Participants accessed the public healthcare system through primary healthcare clinics (PHC). One of the functions of PHCs is prostate-specific antigen (PSA) blood testing to screen for 
prostate cancer in adult men to promote early detection and diagnosis of prostate cancer. After PSA testing, participants were referred through district, regional and tertiary hospitals for further diagnostic testing, imaging and treatment.

- Blood testing in primary health clinics

Participants went to PHCs for general health concerns or because they wanted to take advantage of PSA testing offered by the clinic. Participants mentioned that they were unsure of the reason for PSA testing and the implications of a positive diagnosis. One participant explained that he attended the PHC for his tonsils but underwent PSA testing as part of the visit, and the diagnosis of prostate cancer came as a surprise.

"Why I am here was because of tonsil problems. And then why am I having this (referring to the PSA test that was done) this, tonsils, and then they take my blood, and when everything went back they said everything is negative except, eh, prostate [cancer]." (Participant 9)

Another participant had submitted himself voluntarily for a PSA test. The result was an unexpected cancer diagnosis followed by a rectal examination,

"I didn't know I have this prostate cancer thing but I wanted to know if I was already affected, infected or affected. And then I went to the clinic of the special one (PSA test) for me and send me to the lab [oratory] and then when it came (the results) it wasn't a nice experience because I did not know I was sick. And then after they make tests and all that it's also like feeling it, because mine (the prostate) they told me it is enlarged. So I didn't know how they know that and they said no put this finger at the back (referring to rectal examination) and then they sort of search from [for] it and they felt it and said, no, this is too big." (Participant 5)

- Reasons for being sent between hospitals

Participants were confused by having to go between hospitals to reach the tertiary hospital where their cancer could be treated, for example, with radiation therapy. Participants did not receive enough information explaining the need to go to multiple hospitals. One explained:

"I wanted to know what was the reason I was going here and there. I was told at that time that the reason for coming here (the tertiary-level hospital), you see, you will [would] go there because of this disease. Now I do not know is wrong is wrong or is right." (Participant 6)

Participants also described having to go between hospitals because they could not stay in the hospital where they were receiving radiation therapy. An example of this was given:

"They took me to A (regional-level hospital). There at A (regional-level hospital) they said they had no places for people to sleep. There were no spaces. I had to be admitted to B (tertiary-level hospital). They took me and brought me to B (tertiary-level hospital). There they put me on the machine (radiation treatment machine/linear accelerator), that machine from here at B (tertiary-level hospital). They then took me C (district-level hospital) to admit me there." (Participant 1)

\section{What Does this Mean to My Life and Me?}

Having been diagnosed with prostate cancer, patients had a need to understand how this disease and the treatments would impact their bodies and affect their daily activities.

- What is cancer, and specifically prostate cancer?

Patients need support to come to terms with their cancer diagnosis by understanding what they have. Most participants did not understand what cancer was, let alone specific cancers such as prostate cancer and how the cancer develops.

"It-it is a frightening. But if you are to, you know, what is cancer? You know, just a brief explanation to say, listen, you know, cancer is about this and this. What is this all about?" (Participant 7) and 
"I said how many types of cancers do we have? I didn't know about other cancers. I want to ask you how prostate become cancerous?" (Participant 4)

- Where is it in my body?

The participants wanted to understand where the cancer resided in their bodies. One participant explained in detail how useful he found a diagrammatic explanation of where the tumour was in his body. A healthcare professional explained on a drawing exactly where the tumour was in the body.

"You know, somebody had made a copy for me like this [participant showing a sketch of the anatomical structures in the pelvis]. It's interesting, it starts here according to the information I heard, they said this is the bladder [pointing on the drawing], and somewhere here is above something here. He (the healthcare professional) said the prostate is here [pointing on the drawing]." (Participant 4)

Participants mentioned that they were also curious about what healthcare professionals saw on the imaging examinations. This participant wanted the healthcare professional to share the information from the imaging examinations. Participants also required specific information on the effects of cancer on the body.

"I would like them to explain how they see (referring to the healthcare professional's viewing of radiographic images). How do you feel in your body that you have cancer? How do you see? Those facts. That what and what does what in the body." (Participant 2)

- How serious is it?

For the participants, the word "cancer" conjured up fear and deep concern for life as they know it. More than one participant raised the catch phrase of the "silent killer," Participants needed more information regarding their prognosis and whether their cancer was treatable and curable. For example:

"I was just interested on what was happening with my life. How are they going to help me get right, cure this thing?" (Participant 5)

And another viewed the cancer relative to AIDS:

"Ya, because this thing is a silent killer. Inside you don't feel it... They (the patients) must be told that this thing is [a] killer, it's worse than AIDS or the same as AIDS." (Participant 9)

- Why the treatments?

Patients consented to receiving various treatments for their prostate cancer. Participants mentioned that they were uncertain about what treatments were being offered, why specific treatments were needed and whether the treatments would be effective. Participants expressed the following:

"Sometimes I just wanted to know whether this thing would be, you know, it (the treatment) if this thing is really effective. You know, that I was always wondering what the [treatment] whether that is really effective, you know." (Participant 3)

"Why don't you tell me what I am going to do and what treatment am I going to get? Cause you are the doctor, so you must tell me." (Participant 8)

Another participant had received Zoladex (ADT) multiple times, without understanding what the reason for the treatment was.

"Yes, I think I got it three times. What is it

[Zoladex] for?" (Participant 4)

- What to do and not to do?

Participants expressed a need for information regarding daily living, to regain control of this serious situation. Participants wanted to know how they could support their treatment for the best possible outcomes. One participant asked a wide range of questions, requiring clarity on various levels of self-care:

"Ask for instance-you said you were going for chemotherapy-are there any restrictions? Is there any advice that one can get? Diet maybe? Or exercise? You know, or things like that. What kind of foods to 
avoid, you know, and can I drink? Can I continue to drink? Is it jogging or exercise? You know, that kind. Is it jogging? Or will exercise improve or make the situation worse?" (Participant 7)

\section{The Radiation Treatments}

Having completed a radical course of EBRT for prostate cancer, all the participants required specific information about radiation therapy. Participants mentioned some of the processes that they seemed to understand, but still had many questions about the radiation treatment.

\section{- About the treatment}

Participants expressed the need for in-depth information on what the treatment actually was, and what was expected of them. The participants asked the following questions,

"Why don't you tell me what I am going to do and what treatment am I going to get? Cause you are the doctor, so you must tell me." (Participant 8)

Participants mentioned being uncertain about why they had to fill their bladders on a daily basis before receiving their radiation treatments. This participant explained that he drank water, but did not understand why:

"They made me drink water. I just came from the machine. They gave me a bottle this big [gestures to size of bottle]. I drank it finished. They don't tell me anything. I drink their water and climb onto the machine." (Participant 1)

During radiation treatment, healthcare professionals also need to check the accuracy of the set-up of the radiation treatment fields. Radiation therapists, who deliver the treatment, produce images of the treatment fields whilst the patient is in the treatment position on the treatment couch. Participants do not clearly understand reasons for taking these "X-rays". Participants could not distinguish between the treatment imaging "X-rays" and those taken for diagnostic purposes, and expected an X-ray report, similar to previous experiences in the
X-ray department. This quote highlights the misunderstanding:

"They (the radiation therapists) were taking the X-rays. I wanted to know why [are] they taking X-rays. And they didn't give me results for having the X-ray. Why they were taking those, those, those cassettes?" (Participant 8)

- How will the radiation affect the body? How will the radiation affect the body?

In this setting, participants expressed a need for information on the side effects of radiation treatments.

"After you [I] went through the machine, your [my] urine burns you [me], so if you [I] pass water it burns you [me] a little bit. I was not told that." (Participant 8)

Patients experienced unexpected and unexplained side effects, which left them trying to work out the reasons for the symptoms they experienced. This participant wondered why he was having a "runny tummy" after meals:

"I don't know, my stomach is always loose.

Is [It's] not loose but after eating I must go." (Participant 9)

- What happens next?

Participants expressed feeling uncertain about the future and wanted to know "what next". They wanted to know what other treatments and ongoing medical-related contacts they would need to make. Examples of the questions asked relating to this issue were:

“The next step it's going. I just want them to explain to me how they will go about to the end. I still don't know so far." (Participant 5)

"What, what I want to know now is if I, let's say, I've got only two days to go to finish. After that what's gonna [going to] happen? Do I come for check-ups and that?" (Participant 8)

"That's now [Now that I] am finishing with the radiation, I should [should I] keep on getting that Zoladex?" (Participant 4) 


\section{THEME 2: INFORMATION NEEDS TO ADDRESS THE CONNOTATIONS OF PROSTATE CANCER}

Aside from having specific questions regarding cancer and treatments, participants shared a number of negative experiences and perceptions related to being diagnosed with prostate cancer.

\section{Shame in the Illness}

Participants expressed concern about how they were being perceived by others in the light of their illness. Participants were embarrassed about having cancer because of their own understanding of the connotations of prostate cancer and personal knowledge of how society views cancer. The psychological definition of shame is "a highly unpleasant self-conscious emotion arising from the sense of there being something dishonourable, immodest, or indecorous in one's own conduct or circumstances. It is typically characterized by withdrawal from social intercourse" [25].

- Blame for not understanding

In this study most of the participants were diagnosed with late-stage tumours. Participants mentioned various reasons for presenting with late-stage cancer. One participant did not want to be blamed for not knowing and understanding about cancer and the prostate:

"If now, then they recover [discover] it later, there is nobody to be blame[d] because a patient doesn't know or understand what is happening within himself. When you ask him what is a prostate, he will not understand that there is something in this. That's why I was a bit late to check it, you see." (Participant 4)

while another blamed himself for not understanding:

"I mean, I was totally-totally blank (referring to his understanding of cancer), but I blame myself. Nobody else." (Participant 7)
- Being seen "as a cancer"

Participants mentioned that their communities, and they, were reluctant to talk about cancer due to the negative stigma associated with cancer.

"People at the location (low-income urban settlement) there, if you say you are suffering from prostate [cancer], some of them will say [that] you are cancerous." (Participant 4)

- "Man issues"

A participant wanted to know whether the sexual problems that he was experiencing would resolve after the radiation. The ADT drug Zoladex, which patients receive prior to radiation, would have had an effect on erectile dysfunction. One expressed his concern as follows:

"I would ask them after the treatment would I be able to get my man be back. Aaah well, like I said my manhood is very important first one." (Participant 9)

Men do not readily accept health issues associated with the prostate, because of the link with their masculinity. Participants mentioned a psychological and physical association between the prostate and the effect on "manhood". A participant mentioned witnessing two men refusing treatment because they did not want to experience erectile dysfunction, despite declining health.

"Some of them they are negative. Especially here our clinic in the hospital ' $\mathrm{C}$ ' (district hospital). Two guys they leave their files (abandon their hospital filesrefuse treatment). They said, 'No 1 can't live without my manhood.' Then, they just gone. And I saw him, one of them, after my third week here. [He] is losing weight terribly. I said to him, 'Why do you worry about your manhood while you are very sick like this?' Now you have lost everything. Now he's living while tying his trousers (lost weight). So it's late now for him. I think it's now penetrating the whole body. He doesn't want to come." (Participant 8) 
Participants mentioned that some men avoided prostate screening, because they felt screening was unnecessary and there was nothing physically wrong with them. One participant explained,

"They (other men) say: "No, l don't have anything. Why should 1 go there (to the clinic/hospital)?" They do like me, saying '[I] am health[y]. There is nothing wrong with me', and it's true, l didn't feel anything." (Participant 5)

\section{Message for Other Men}

Following treatment, participants expressed that it was important to raise awareness about prostate cancer screening and treatment.

- Getting tested

Some participants took it upon themselves to encourage male members of their family, friends and community to go for testing for the disease. Participants stressed the danger of prostate cancer and the importance of testing, because of the hidden nature of the illness. This was encapsulated in this quote:

"l think we need to be educated seriously about this thing (prostate cancer), because as far as 1 know, the doctors told me that it's a silent killer. That's very important, because it is a very dangerous disease, especially with us men. You know men, sometimes you tell somebody [to] go for tests. Because this thing (the prostate cancer), you won't know if it's there or not." (Participant 5)

\section{DISCUSSION}

The information needs of prostate cancer patients have been quantitatively assessed in first-world settings $[12,13,22]$. In these settings, patients have shown a universal need for information revolving around the broad questions of "What is prostate cancer?", "How does the treatment work?", "What lifestyle should be followed?", "What food and nutrition is suggested?", "What are the side effects of treatment" and "What is the follow-up after treatment?". The patients in this study asked similar questions, but required further explanation and illustration of the position of the prostate within the body.

In this study, participants highlighted a need to change perceptions regarding prostate cancer. Changing negative perceptions in participants' communities should serve as a touchstone to guide further education in local societies. Participants all agreed on the importance of creating prostate cancer awareness and the need for prostate screening among men in their neighbourhoods. In South Africa, health promotion campaign groups such as the Cancer Association of South Africa (CANSA) and the Prostate Cancer Foundation of South Africa provide information on prostate cancer and screening. Participants indicated that information campaigns should emphasise the importance of being tested for prostate cancer, even if men are feeling well, as this is a hidden disease. Participants also stressed that screening should be accompanied by pre- and post-screening counselling for patients. Counselling sessions will help men understand the reasons for screening and prepare them for outcomes. The European Association of Urology strongly recommends that all prostate screening be accompanied by prior counselling with information on the potential risks and benefits [26]. This aspect of prostate screening needs to be strengthened in the healthcare services where testing is done.

In the South African context, patients have to traverse a fragmented healthcare service to access their examinations and treatments [27]. This creates both confusion and uncertainty for patients. Patients require personalised information about the healthcare facilities they will need to visit and the purpose for having to visit each facility. Within these healthcare facilities, prostate cancer is managed with a multimodal treatment approach. During medical consultations, patients need to receive a cohesive picture of the treatment plan, the timing, duration and purpose of the treatments, an explanation of how the treatments work and information on the expected side effects. Importantly, clear protocols need to be developed, stating who is 
responsible for providing which information to the patient, when the patient is attending different domains of the cancer care continuum [28].

Once a patient is diagnosed with prostate cancer, patients have a need to know, "how serious is it?" Amongst the participants in this study, this suggests that the sensitive issue of prognosis is not being fully addressed. This aspect of interaction between the medical healthcare professional and the patient during initial diagnosis and treatment requires attention [10]. This discussion is critical for the patient to understand and come to terms with the uncertainty of the cancer. Participants used the metaphor "the silent killer" when sharing cancer-related information, conjuring negative connotations of the disease. Healthcare professionals should be mindful and find ways to address the hidden self-blame that being diagnosed with cancer engenders in the patient [29].

Being diagnosed with prostate cancer also triggers certain negative connotations in communities that emanate from local perceptions of cancer. For these patients, the reality of being viewed "as a cancer" and the taboo of the nature of masculinity overlies an unspoken shame. The social stigma regarding prostate cancer observed in this study is similar to the findings in a qualitative study exploring men's perceptions, beliefs and practices for prostate screening in Cameroon [30]. Providing information to overcome internalised and community-entrenched stigmas is challenging [31]. Organisations such as CANSA face challenges in addressing the issue of stigma within cancer awareness campaigns.

The treatments, and in particular radiation treatments, create another set of information needs. The radiation therapist is primarily responsible for providing this information from a patient-centred perspective [32]. Patients need to be warned about potential side effects of the radiation treatments and an explanation of the symptoms that they might be experiencing [33]. However, the information provided needs to take into account the disease and other treatments that patients have received or are receiving. For example, participants indicated that they had experienced diarrhoea, burning upon micturition and erectile dysfunction while they were receiving EBRT. In explaining and providing information to the patients, radiation therapists need to address issues such as the fact that diarrhoea can be worse if EBRT is combined with ADT, and burning upon micturition might be exacerbated by the extent of the prostatic tumour [34]. Although the patients' erectile dysfunction might be due to the cancer and/or the ADT-and not the EBRT - the patients require information reinforcement by the radiation therapists [34]. Advice on how they can care for themselves in the event that these symptoms occur, and adjusting lifestyles to cope, should be an important component of the information provided. The radiation therapist should explain why the radiation treatment is needed, what will happen during the treatment and what is expected of the patient in the process. Therapists should pay particular attention to the pretreatment "water-drinking routine" and the radiographic imaging performed during the treatments [33]. Clear explanations of what will happen after the completion of radiation treatments as regards further treatment and expected medical contacts should be provided.

\section{Limitations}

The interviews were conducted only at a single point in time. Therefore, it cannot be assumed that a patient's information needs were always consistent with the study results, since they may have differed depending on the phase of the cancer care continuum that they were in. The data collection was performed at only one research setting, and therefore the transferability of the study results is limited. The patients were the only ones interviewed, and the viewpoints of healthcare providers regarding information-giving were not considered. Unintentionally, only black men were interviewed and no data exists on the information needs of South African white men diagnosed with prostate cancer. Therefore, no comparison could be made between the information needs of patients from different cultures and races in South Africa. 


\section{CONCLUSION}

This study qualitatively assessed the information needs of men diagnosed with prostate cancer. Patients have a wide range of information needs pertaining to their understanding of what the cancer is, what is happening to them, what they should do and the reasons for having to go through various processes and treatments. Understanding where to go and what will happen in the different parts of the healthcare system with regard to the diagnosis and treatment of the prostate cancer is also needed. This study further highlights that information needs go beyond the individual and must extend to local communities. Particular attention needs to be given to lower-income communities, where residents are not proficient in English, as most awareness campaigns are currently presented in English. Increasing awareness about the importance of screening will address the life-world needs of patients when they are diagnosed with prostate cancer.

\section{ACKNOWLEDGEMENTS}

The authors wish to thank all the patients for the insights that they provided within the interviews in the study.

Funding. No funding or sponsorship was received for this study or publication of this article.

Editorial Assistance. Dr C. A. Tosh of the University of Pretoria provided editorial assistance. No funding was received for this assistance.

Authorship. All named authors take responsibility for the integrity of the work as a whole, and have given their approval for this version to be published.

Authorship Contributions. Prudence Kawinga, Aviwe Makenzi and Melissa Taljaard were responsible for conducting the patient interviews. Aviwe Makenzi was responsible for translating the Zulu language patient responses into English. Germaine Lovric, Melissa Taljaard, Aviwe Makenzi and Prudence Kawinga were responsible for the data analysis. Germaine Lovric and Melissa Taljaard were responsible for writing and reviewing the article.

Disclosures. Melissa Taljaard, Germaine T. Lovric, Aviwe M. Makenzi and Prudence Kawinga have nothing to disclose.

Compliance with Ethics Guidelines. The National Health Research Department gave permission to interview the patients in this public hospital. The University of Pretoria Health Sciences Ethics Committee approved the study, with Ethics Reference No. 602/2018. Informed consent was obtained from all participants, and the study was conducted in accordance with the Helsinki Declaration.

Data Availability. The data sets generated during and/or analyzed during the current study are available from the corresponding author on reasonable request.

Open Access. This article is licensed under a Creative Commons Attribution-NonCommercial 4.0 International License, which permits any non-commercial use, sharing, adaptation, distribution and reproduction in any medium or format, as long as you give appropriate credit to the original author(s) and the source, provide a link to the Creative Commons licence, and indicate if changes were made. The images or other third party material in this article are included in the article's Creative Commons licence, unless indicated otherwise in a credit line to the material. If material is not included in the article's Creative Commons licence and your intended use is not permitted by statutory regulation or exceeds the permitted use, you will need to obtain permission directly from the copyright holder. To view a copy of this licence, visit http:// creativecommons.org/licenses/by-nc/4.0/. 


\section{REFERENCES}

1. Bray F, Ferlay J, Soerjomataram I, Siegel R, Torre L, Jemal A. Global cancer statistics 2018: GLOBOCAN estimates of incidence and mortality worldwide for 36 cancers in 185 countries. CA Cancer J Clin. 2018;68(6):394-424. https://doi.org/10.3322/caac. 21492.

2. Hayes VM, Bornman MSR. Prostate cancer in Southern Africa: does Africa hold untapped potential to add value to the current understanding of a common disease? J Glob Oncol. 2017;4:1-7.

3. Sherriff A, Da Costa N, Engelbrecht A, Li A, Price N, Joubert G. Prostate cancer profile and risk stratification of patients treated at Universitas Annex Department of Oncology, Bloemfontein, Free State, during 2008 to 2010. S Afr Fam Pract. 2015;57(4): 247-52.

4. Dewar M, Kaestner L, Zikhali Q, Jehle K, Sinha S, Lazarus J. Investigating racial differences in clinical and pathological features of prostate cancer in South African men. S Afr J Surg. 2018;56(2):54-8.

5. Department of Health Republic of South Africa. White paper-National Health Act, 2003-National Health Insurance policy to universal coverage. 2017. [cited 2020 June 4]. https://www.health.gov. za/index.php/nhi-documents?download=2257: whitepaper-nhi-2017.

6. Department of Health Republic of South Africa. 2016 Annexure B-Regional, Tertiary and Central Hospital Services. [cited 2020 June 4]. https://www. health.gov.za/index.php/nhi/category/267-nhi-016? download=1142: annexure-b-tertiary-services-definitions-package-of-care-december-2015.

7. South Africa. Department of Health. National Health Act, No. 35101, 2003. Regulations relating to categories of hospitals. Gazette no. R.185. 2012

8. Washington C, Leaver D. Principles and practice of radiation therapy. 4th ed. St. Louis: Elsevier; 2016.

9. Christalle E, Zill JM, Frerichs W, Härter M, Nestoriuc $Y$, Dirmaier J, et al. Assessment of patient information needs: a systematic review of measures. PLoS ONE. 2019. https://doi.org/10.1371/journal. pone.0209165.

10. Epstein RM, Street RL. Patient centered communication in cancer care-promoting healing and reducing suffering. Bethesda: NIH Publication; 2007.

11. King AJL, Evans M, Moore THM, Paterson C, Sharp D, Persad R, Huntley AL. Prostate cancer and supportive care: a systematic review and qualitative synthesis of men's experiences and unmet needs. Eur J Cancer Care. 2015;24(5):618-34. https://doi. org/10.1111/ecc.12286.

12. Dale J, Jatsch W, Hughes N, Pearce A, Meystre C. Information needs and prostate cancer: the development of a systematic means of identification. BJU Int. 2004;94(1):63-9.

13. Rüesch $P$, Schaffert R, Fischer S, Feldman-Stewart D, Ruszat R, Spörri P, et al. Information needs of earlystage prostate cancer patients: within- and betweengroup agreement of patients and health professionals. Support Care Cancer. 2014;22:999-1007. https://doi.org/10.1007/s00520-013-2052-8.

14. Chauhan M, Holch P, Holborn C. Assessing the information and support needs of radical prostate cancer patients and acceptability of a group-based treatment review: a questionnaire and qualitative interview study. J Radiother Prac. 2018;17(2): 151-61. https://doi.org/10.1017/S1460396917000 644.

15. Kassianos AP, Raats MM, Gage H. An exploratory study on the information needs of prostate cancer patients and their partners. Health Psychol Res. 2016;4:4786-93. https://doi.org/10.4081/hpr.2016. 4786.

16. Feldman-Stewart D, Capirci C, Brennenstuhl C, Tong C, Abacioglu U, Gawkowska-Suwinska M, et al. Information needs of early-stage prostate cancer patients: a comparison of nine countries. Radiother Oncol. 2010;94(3):328-33. https://doi. org/10.1016/j.radonc.2009.12.038.

17. Gopal RL, Beaver K, Barnett T, Ismail NS. A comparison of the information needs of women newly diagnosed with breast cancer in Malaysia and the United Kingdom. Cancer Nurs. 2005;28(2):132-40.

18. Schinkel S, Schouten BC, van Weert JC. Are GP patients' needs being met? Unfulfilled information needs among native-Dutch and Turkish-Dutch patients. Patient Educ Couns. 2013;90(2):261-7.

19. Lovric G, Makanjee C. A qualitative study exploring patients' expectations and experiences of the localization event as part of radiation therapy. J Radiol Nurs. 2018;37:205-10.

20. Caell K, Ray L, Mill J. "Clear as mud": toward greater clarity in generic qualitative research. Int J Qual Methods. 2003;2(2):1-23.

21. Sandelowski M. What's in a name? Qualitative description revisited. Res Nurs Health. 2010;33(1): 77-84. https://doi.org/10.1002/nur.20362. 
22. Morse JM, Field PA. Qualitative research methods for health professionals. Thousands Oaks: Sage Publications; 1995.

23. Elo S, Kyngäs $H$. The qualitative content analysis process. J Adv Nurs. 2008;62(1):107-15. https://doi. org/10.1111/j.1365-2648.2007.04569.x.

24. Saldana J. The coding manual for qualitative researchers. London: Sage publishers; 2009.

25. American Psychology Association [Internet] AP Dictionary of Psychology [updated:cited]. Accessed from: https://dictionary.apa.org.

26. Gandaglia G, Albers P, Abrahamsson PA, Brigantia A, Cattoe JWF, Chapple CR, et al. Structured population-based prostate-specific antigen screening for prostate cancer: the European Association of Urology position in 2019. Eur Urol. 2019;76(2): 142-50. https://doi.org/10.1016/j.eururo.2019.04. 033.

27. Basu S, Andrews J, Kishore S, Panjabi R, Stuckler D. Comparative performance of private and public healthcare systems in low- and middle-income countries: a systematic review. PLoS Med. 2012;9(6):e1001244. https://doi.org/10.1371/ journal.pmed.1001244.

28. Levit L, Balogh E, Nass S, Ganz PA, editors. Delivering high-quality cancer care: Charting a new course for a system in crisis. Washington DC: National Academic Press; 2013.

29. Phelan SM, Griffin JM, Jackson GL, Zafar SY, Hellerstedt W, Stahre M, et al. Stigma, perceived blame, self-blame, and depressive symptoms in men with colorectal cancer. Psychooncology. 2013;22(1):65-73. https://doi.org/10.1002/pon. 2048.

30. Kaninjing E, Lopez I, Nguyen J, Odedina F, Young ME. Prostate cancer screening perception, beliefs, and practices among men in Bamenda, Cameroon. Am J Mens Health. 2018;12(5):1463-72. https://doi. org/10.1177/1557988318768596.

31. Stangl AL, Earnshaw VA, Logie $\mathrm{CH}$, van Brakel W, Simbay L, Barré I, et al. The Health Stigma and Discrimination Framework: a global, crosscutting framework to inform research, intervention development, and policy on health-related stigmas. BMC Med. 2019;17(1):31. https://doi.org/10.1186/ s12916-019-1271-3.

32. Zaorsky NG, Showalter TN, Ezzell GA, Nguyen PL, Assimos DG, D'Amico AV, et al. ACR Appropriateness Criteria external beam radiation therapy treatment planning for clinically localized prostate cancer, part I of II. Adv Radiat Oncol. 2017;2(3): 437-54. https://doi.org/10.1016/j.adro.2017.03. 003.

33. Symonds RP, Deehan C, Meredith C, Mills JA, editors. Walter and Miller's textbook of radiotherapy: radiation physics, therapy and oncology. 7th ed. Edinburgh: Churchill Livingstone; 2012. p. 602.

34. Drudge-Coates L, Oh WK, Tombal B, Delacruz A, Tomlinson B, Ripley AV, et al. Recognizing symptom burden in advanced prostate cancer: a global patient and caregiver survey. Clin Genitourin Cancer. 2017;16(2):e411-e419419. 